\title{
Review of Real-Time Release Testing of Pharmaceutical Tablets: State-of-the Art, Challenges and Future Perspective
}

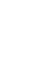

4

${ }^{\mathrm{f} G S K}$ Research \& Development, Park Road, Ware, SG12 ODP, UK
${ }^{g}$ Department of Chemical Engineering and Biotechnology, University of Cambridge, Philippa Fawcett Drive, CB3 OAS, Cambridge, UK

*corresponding author:

Electronic address: daniel.markl@strath.ac.uk

Phone: $\quad+44(0) 1414447115$

Address: $\quad$ CMAC Future Manufacturing Research Hub

University of Strathclyde

Technology and Innovation Centre

Level 6

99 George Street

Glasgow G1 1RD 


\section{ABSTRACT}

In the last decade significant advances have been made in process analytical technologies and digital manufacturing of pharmaceutical oral solid dosage forms leading to enhanced product knowledge and process understanding. These developments provide an excellent platform for realising real-time release testing (RTRT) to eliminate all, or certain, off-line end product tests assuring that the drug product is of intended quality. This review article presents the state of the art, an RTRT development workflow as well as challenges and opportunities of RTRT in batch and continuous manufacturing of pharmaceutical tablets. Critical quality attributes, regulatory aspects and the scientific basis of enabling technologies and models for RTRT are discussed and a systematic development workflow for the robust design of an RTRT environment is presented. This includes the discussion of key considerations for the identification of the critical quality attributes and points of testing as well as the development of the sampling strategy, a hard and/or soft sensor approach and operational procedures. The final sections present two RTRT use cases in an industrial setting as well as critically discuss challenges and provide a future perspective of RTRT.

Keywords: Real-time release testing, tablet manufacturing, process analytical technology, soft sensors, models

\section{Introduction}

The majority of medicines are administered to a patient by oral solid dosage forms (tablets and capsules), where an active pharmaceutical ingredient (API) is combined with a range of excipients through a sequence of processes that improve manufacturability, ease of use and the therapeutic effect. Throughout this manufacturing process, the safety and efficacy of the drug product are maintained through a control strategy. For this to work, it is paramount to detect, control and monitor any sources of material and process variations which impact the critical quality attributes (CQAs) of the drug product.

Given the complex nature of the formulations and processes, a full scientific understanding of all aspects that affect the drug product quality is generally not possible and hence frequent in-process control (IPC) measurements of process parameters and quality attributes of intermediate materials as well as the final product are essential steps to assure the quality of the drug product. This approach is not only relevant for batch processing, but it is of particular importance also in the context of continuous manufacturing (CM) to establish a state of control (Lee et al., 2015). As part of the progress in sensor technology over the last decade, a range of lab-based techniques have advanced into industrially robust and reliable process analysers that are suitable for process analytical technology (PAT) applications (Johansson et al., 2010, Fonteyne et al., 2015; Laske et al., 2017). These technological advancements coupled with the significant progress in process modelling and soft sensors opened up new opportunities to access a wide range of chemical and physical material characteristics during manufacturing. 
Once a robust formulation and process are developed, real-time release testing (RTRT) can be implemented to evaluate and ensure the quality based on process data by combining process controls and measured material attributes of one or all CQAs. RTRT is defined as "the ability to evaluate and ensure the quality of in-process and/or final product based on process data, which typically include a valid combination of measured material attributes and process controls." (ICH, 2009). Apart from assuring product quality, this approach is aimed at reducing labour and cost-intensive off-line product release testing. From an industrial point of view, the effort on developing and deploying hard (PAT) and/or soft sensors falls typically into the responsibilities of a development organisation, while the beneficiary is usually the manufacturing and quality organisation. RTRT is thus the most direct monetary return (i.e., financial incentive) of generating a sound process understanding as well as developing and deploying sensors to measure CQAs.

This review aims to provide an overview of RTRT as part of a control strategy (Section 2) and highlight the CQAs and measurement techniques (Section 3) relevant for RTRT in a tablet batch and continuous manufacturing line. Many CQAs cannot be measured directly and thus need to be predicted using models and one or several measured material properties and/or process parameters. Section 4 thus discusses the development, implementation and life cycle maintenance of models in the context of RTRT. A RTRT development workflow is presented in Section 5, which highlights key considerations in developing soft and hard sensors as well as identifying the points of testing and sampling plan. The final two sections present two industrial use cases (Section 6) and provide a critical review of challenges and a future perspective of RTRT (Section 7).

\section{Real-time Release Testing as Part of a Pharmaceutical Control Strategy}

RTRT is an integral part of a control strategy, where the controls can include "parameters and attributes related to drug substance and drug product materials and components, facility and equipment operating conditions, in-process controls, finished product specifications, and the associated methods and frequency of monitoring and control" (ICH, 2007). A pharmaceutical control strategy can typically be associated to one of the three categories, as defined by Yu et al. (2014), from the lowest level based on recipe control and end product testing (Level 3 ) to the highest level (Level 1 ) of control enabling fully automated processing. Descriptions of these different categories are provided in Appendix B. Regardless of the category, a control strategy includes a specification of the methods used to control drug substance and product properties. Methods used to make quality-based control decisions during RTRT are referred to as primary methods and considered as high (impact) risk methods according to the risk impact matrix as described in 'ICH Quality Implementation Working Group Points to Consider (R2)' (ICH, 2011, 1995). In other words, high risk methods are used to determine CQAs, that are not subsequently tested 
downstream. A medium (impact) risk method, in contrast, is used to control an attribute which is verified downstream, these methods are considered as medium (impact) risk, which have a lower validation burden. Methods that are not used to make control decisions are regarded as low (impact) risk and, whilst the performance of the equipment or method needs to be tested to ensure it is fit for purpose, often method validation is less rigorous.

Although the approved control strategy describes the steps that are required to ensure the process stays in a state of control, it is possible to report the compliance to these requirements as justification for product disposition under RTRT. Acceptance limits for RTRT should also include minimum sample sizes and criteria for proceeding at risk when individual data points are missing.

\section{Critical Quality Attributes}

CQAs of pharmaceutical tablets are commonly material properties affecting product purity, strength, drug release and/or stability. In many RTRT applications one or several measured material properties are used to predict the CQA of interest with a validated model (see Section 4 for more details). The process flow diagram in Figure 1 indicates the most prevalent CQAs in batch and continuous tablet manufacturing for the three common processing routes: 1) direct compression, 2) dry granulation and 3) wet granulation. Compared to Option 1, directly compressed tablets, Options 2 and 3 are very common in an industrial setting as a granulation step can improve flow properties and compression properties and thus enable a higher drug load and improved content uniformity for low dose formulation products. Although the CQAs in Figure 1 are relevant for both batch and CM of tablets, the selection of the sensor type and its integration as well as the point of testing and sampling plan might vary between the two manufacturing configurations. Sensors in CM may also be used as an in-process control to divert material to waste when the monitored attribute is outside the control limit (e.g. when tablet content uniformity limit is not achieved), whereas in batch manufacturing the measurements are commonly used to detect the process end point (e.g. stopping the coating process when the desired coating thickness is reached).

It is important to emphasise at this point that existing RTRT's for the discussed CQA's are not feasible for all drug product manufacturing processes and need to be evaluated on a case by case basis. The suitability of a CQA for a given drug substance and manufacturing setting needs to be assessed during the development of the RTRT method as discussed in Section 5. When choosing the point of testing and type of sensor for RTRT, accuracy, speed, robustness, repeatability, selectivity and sampling strategy needs to be considered in a risk and cost-benefit analysis (more details are provided in Section 5). This analysis should also address whether a direct measurement of the property is possible, or a multivariate model is needed to predict the CQA from one or several measurement signals and/or material attributes. This is indicated in Figure 2 for each CQA and measurement technology discussed below. In this context, a direct 
measurement is the determination of the size or amount of a property of interest using a sensor without the need of a statistical model.

This section discusses relevant CQAs and hard (PAT) and/or soft sensors in use to measure them. Key information of RTRT implementations and other relevant PAT applications are summarised in Table C.1 in Appendix C. However, further detail about the various PAT methods is outside of the scope of this article and the interested reader is referred to other review articles (Johansson et al., 2010; Fonteyne et al., 2015; Laske et al., 2017). In the current literature many of the described applications used NIR or Raman spectroscopy. The choice between those two methods typically depends on the absorption features of API and excipients in the respective spectral range as well as the required speed of analysis. Selection criteria and in-depth discussion of these methods can be found in a range of publications (Beer et al., 2011; Nagy et al., 2018; Paudel et al., 2015; Reich, 2005; Roggo et al., 2007).

\subsection{Blend Mixing Homogeneity and Tablet Content Uniformity}

Content uniformity ensures that a consistent dose of the drug is maintained between tablets and batches. In a tablet, it is primarily influenced by the homogeneity of the powder mixture after the final blending step and the tablet weight. Blending aims to produce a homogeneous mixture of drug and excipient particles that accurately represents the desired ratios of the components. The mixing homogeneity is commonly monitored in batch blending to detect the end point of the mixing (Berntsson et al., 2002; Crouter and Briens, 2019; Pestieau et al., 2014; Sibik et al., 2017). For continuous processes, the mixing homogeneity is assessed of a continuous stream of powder, which enables feedback process control as well as in-process control to divert material to waste. In the continuous case, it is more appropriate to integrate a PAT method in the transfer line between a blender and the tablet press (Järvinen et al., 2013; Palmer et al., 2018) or the feed frame of the tablet press (Hetrick et al., 2017), as discussed by Almaya et al. (2017). If the point of testing is in the transfer line, then a sampling device that presents the sample (i.e. powder) in a consistent and representative manner to the sensor probe is required. A critical aspect of the integration of PAT systems in transfer lines and also in the processes is the use of suitable sampling devices and their configuration as discussed in Section 5. For example, several research groups and pharmaceutical companies have integrated a NIR probe in the feed frame of a tablet press to monitor mixing homogeneity in close proximity of the subsequent compression step so that the measurement represents the composition of the tablets as closely as possible (Manley and Shi, 2018a). In most RTRT applications the mixing homogeneity is measured using NIR or Raman spectroscopy and sampling is performed in a reflection setup.

Once tablets are formed during compression, the tablet content uniformity can also be determined using a spectroscopic measurement. For such applications, NIR and Raman spectroscopy measurements are 
159

160

161

162

163

164

165

166

167

168

169

170

171

172

173

174

175

176

177

178

179

180

181

182

183

184

185

186

187

188

189

190

191

also commonly employed. The quantification of the drug substance content and its uniformity in pharmaceutical tablets is well established as an at-line, on-line and even in-line setting (Harms et al., 2019; Hetrick et al., 2017; Järvinen et al., 2013). In contrast to traditional content and uniformity testing (e.g. HPLC and UV), PAT methods can be applied to analyse intermediate materials and final products during manufacturing within seconds and without the need for sample preparation.

Goodwin et al. (2018) demonstrated an RTRT implementation of NIR spectroscopy to determine drug content and uniformity of tablets prepared by a batch manufacturing process including high shear wet granulation. The authors evaluated the suitability of three options to analyse the content uniformity for RTRT: i) individual tablet weight data, ii) estimated individual tablet content data based on weight variations, and iii) individual tablet content by NIR. For their application they selected option iii) as it did not rely on assumptions such as the concentration of the drug in the tablet is at target. Goodwin et al. stressed that the sample size (i.e. number of analysed tablets) was limited by the acquisition rate of the spectrometer and the time and accuracy of the positioning of the sample for the measurement. This can be overcome by integrating the NIR probe directly in the tablet press (Järvinen et al., 2013; Karande et al., 2010). For example, Järvinen et al. (2013) demonstrated the implementation of the drug content measurement at an acquisition rate of 125,000 tablets/ $h$ at the tablet press.

Recently, the compression force was used to predict the weight uniformity and/or content uniformity of tablets as an indirect measure of the tablet content uniformity (Manley et al., 2019; Manley and Shi, 2018) - more details are discussed in Section 6.2. This soft sensor allowed the authors to implement an in-line quality control strategy that makes product collection decisions on $100 \%$ of the tablets using a multichannel discharge chute.

\subsection{Moisture Content}

Moisture content can be classified as a CQA in a tablet manufacturing line that involves wet granulation. In wet granulation, a liquid (e.g. pure water, EtOH, IPA or a suspension) is added to a dry powder mix to develop liquid bridges between particles and eventually form granules that improve flowability, homogeneity and compressibility of the raw materials (Kleinebudde, 2004; Vervaet and Remon, 2005). The wet granules are subsequently dried to reach a desired residual moisture content, and in turn density/porosity, before compaction. This residual moisture content may impact mechanical properties (i.e. tensile strength) (Ahlneck and Alderborn, 1989; Hartung et al., 2010), dissolution performance (Gabbott et al., 2016) as well as chemical and physical stability (Hausman et al., 2005; Viljoen et al., 2013) of the solid dosage form. Moisture content can be tested at different points in the process. In the majority of cases, the moisture content is assessed during drying (Buschmüller et al., 2008; Hausman et al., 2005) in order to determine the endpoint of drying. It has also been measured during (Hartung et al., 2010) or 
192

193

194

195

196

197

198

199

200

201

202

203

204

205

206

207

208

209

210

211

212

213

214

215

216

217

218

219

220

221

222

223

after wet granulation (Fonteyne et al., 2012) as well as during milling (Chablani et al., 2011) and after roller compaction (Gupta et al., 2005). In many cases the availability of integration ports for the sensor in the process drives the selection procedure of the point of testing.

NIR and microwave resonance technology (MRT) are the most popular methods to quantify the residual moisture content. MRT was demonstrated to monitor the moisture content during fluid-bed drying (Austin et al., 2013; Buschmüller et al., 2008; Lourenço et al., 2011). The low operating frequency of MRT allows electromagnetic radiation to penetrate several centimetres into the material. In contrast, the penetration depth of NIR spectroscopy is limited to a few millimetres. The MRT signal thus reflects the moisture content of a significantly larger sample volume, which is a crucial parameter to consider when selecting an appropriate RTRT technology, as discussed in Section 5. Although MRT can outperform NIR spectroscopy in terms of determining the moisture content, NIR spectroscopy has benefits in its versatility as it can be used to monitor the chemical composition of a powder blend more accurately than MRT (Austin et al., 2013).

\subsection{Solid State Chemistry}

It is critical to identify process-induced solid state transformations as a change in its form (polymorphs, hydrates and solvates) can directly impact the solubility, stability and bioavailability of the drug. A solid state transformation can be induced by a change in temperature, pressure or humidity during processing.

Wet granulation is considered as one of the most critical steps in terms of inducing a solid state transformation of the API. NIR and Raman spectroscopy is commonly integrated in the granulation or subsequent drying process to identify changes in the solid state. Wikström et al. (2004) deployed Raman spectroscopy to in-line monitor the hydrate formation of theophylline in a high-shear wet granulator. The dehydration of erythromycin was observed in-line during fluid-bed drying using NIR spectroscopy (Römer et al., 2008). Fonteyne et al. (2012b) monitored the solid state of granules at the outlet of a continuous twin screw granulation process and they highlighted that the differentiation between theophylline polymorphic transformations were more pronounced in Raman spectra than in NIR spectra.

\subsection{Dissolution}

Dissolution testing is an integral part of the regulatory filing worldwide, and it plays a vital role in the drug product development lifecycle ranging from process development, validation, evaluation of post-approval formulation changes to drug product quality, assessment of bioequivalence, and as a surrogate for in vivo drug release (Zaborenko et al., 2019). In vitro dissolution testing is used routinely for batch release testing to detect CQA changes affecting in vivo release of the drug product. The white paper by Zaborenko et al. (2019) provides a general strategy for predictive in vitro dissolution model development. 
Prediction of tablet dissolution performance is possible using a surrogate model (see Section 5 for more details) that is capable of calculating a dissolution property (e.g. drug content percentage release after $15 \mathrm{~min}$ ) from material properties and/or measurable quality attributes. The most commonly measured quality attributes are tablet strength, weight, thickness, porosity as well as coating thickness for direct compression formulations (Option 1 in Figure 1). In addition to these attributes, granule size and density need to be considered in case of a granulated product (Option 2 and 3 in Figure 1). Several groups have developed models for predicting a dissolution characteristic from individual (Hernandez et al., 2016; Pawar et al., 2016; Zannikos et al., 1991) and a combination (Nagy et al., 2019; Otsuka et al., 2007) of NIR and Raman spectroscopic measurements in reflection as well as in transmission.

\subsubsection{Granule Size and Density}

The size and the density (or porosity) of granules produced in wet or dry granulation impact the disintegration and dissolution of a tablet when it comes in contact with physiological fluid. During the disintegration, a tablet tends to break up into its granules. The specific surface area and the density of the granules drive the liquid uptake of the granules and their break up into the primary drug particles (Markl and Zeitler, 2017). Both granule size and density can impact drug product performance and its effect on dissolution varies depending on the formulation, process setting and API. As an example, van den Ban and Goodwin (2017) discussed a drug product where the granule density has a stronger impact than granule size on product performance. Although granule density is frequently identified as a CQA, an accurate, fast and non-destructive determination of this granule property is challenging. Narang et al. (2015) demonstrated an in-line approach for a high shear wet granulator that utilises a drag force flow (DFF) sensor to measure a signal reflecting both granule size and density changes. It was shown that the change of ribbon density after dry granulation can be monitored using an in-line microwave resonance sensor (Austin et al., 2013), Gas in Scattering Media Absorption Spectroscopy (GASMAS; Sjöholm et al, 2001, Svensson et al, 2007, Johansson et al, 2020) or in-line NIR spectroscopy coupled with a principal component analysis (PCA) and partial-least squares (PLS) regression (Acevedo et al., 2012).In terms of granule size, measurement techniques are more advanced than for granule density. The granule size has been measured in-line during or after granulation using a range of different techniques including spatial filter velocimetry (SFV) (Fonteyne et al., 2012a), focused beam reflectance measurements (FBRM) (Kumar et al., 2013), microwave resonance technology (MRT) (Lourenço et al., 2011), acoustic emission (AE) (Whitaker et al., 2000), photometric imaging technique (PIT) (Fonteyne et al., 2012a) and NIR spectroscopy (Khorasani et al., 2016). A different approach was demonstrated by Närvänen et al. (2009) for fluid-bed granulation, who developed a soft sensor that was capable of predicting the granule size from measured and derived process data. In this approach, they used 41 parameters in total ranging from 
257

the temperature of the process room and granulation chamber to the flow rate of inlet air and cumulative enthalpy of the water vapour in outlet air.

\subsubsection{Tablet Strength, Weight and Thickness}

Tablet strength (e.g. hardness, breaking force, crushing strength or tensile strength) is a measure of the strength of interparticle bonds formed during granulation and compaction. These particle-particle bonds need to be ruptured during tablet disintegration which makes the tablet strength a crucial property that affects the dissolution. Moreover, tablet hardness, thickness, and tablet size are parameters that are commonly measured at-line after compaction using an automated testing system and can be integrated into spectroscopic sensor systems. There are also some articles demonstrating the use of NIR spectroscopy (Donoso et al., 2003; Luukkonen et al., 2008) and acoustic emission (Razavi et al., 2016) to predict the tablet strength.

\subsubsection{Tablet Porosity}

Porosity is a fundamental tablet property that impacts mechanical attributes (tensile strength) and tablet dissolution performance. It particularly affects the rate at which the tablet takes up physiological fluids during dissolution, which is the first and often rate-limiting step during tablet disintegration (Markl et al., 2018; Markl and Zeitler, 2017).

Traditionally, tablet porosity ( 1 - solid fraction) is calculated using the tablet weight, tablet thickness and tooling geometry:

$f=1-\varrho_{\mathrm{d}}=1-\frac{m}{V \varrho_{\mathrm{t}}}$

Eq. 1

with $\varrho_{\mathrm{d}}$ as the relative density or solid fraction, $m$ as the mass, $V$ as the volume of the tablet and $\varrho_{\mathrm{t}}$ as the true density of the solid material. Eq. 1 can be further refined for a given set of tablet tooling by

$f=1-\frac{m}{\left(2 V_{\text {cup }}+\left[A_{\text {die }}\left(t-2 d_{\text {cup }}\right)\right]\right) \varrho_{\mathrm{t}}}$

with the tablet tooling parameters $V_{\text {cup }}, d_{\text {cup }}$ and $A_{\text {die }}$ as the volume of the cup, depth of the cup and diehole area, respectively. The true density value is commonly measured using helium pycnometry or calculated by considering the weight percentage of true density values of the individual components.

Recently, it was demonstrated that tablet porosity could be measured in a non-destructive and contactless manner using terahertz time-domain spectroscopy (Markl et al., 2018b; Markl and Zeitler, 2017). It was also shown that this method is capable of predicting the disintegration time and dissolution characteristics for granulated products (Markl et al., 2017b, 2018a). 


\subsubsection{Coating Thickness}

287

288

289

290

291

292

293

294

295

296

297

298

299

300

301

302

303

304

305

306

307

308

309

310

311

312

313

314

315

316

317

318

The coating thickness influences the drug release process in particular in the case of functional coatings, which are designed to act as a diffusion barrier or to protect the API against degradation in the stomach (enteric coatings). Both functions highly depend on the layer thickness and its uniformity, equally for single tablets (intra-tablet coating variability) as well as between tablets (inter-tablet coating variability).

The coating thickness can be measured indirectly using spectroscopic methods (NIR and Raman spectroscopy) by analysing the attenuation of spectral features of one of the mutually exclusive constituents in the product substrate or in the coating formulation (Müller et al., 2012; Pérez-Ramos et al., 2005; Tabasi et al., 2008; Wirges et al., 2013). This typically requires the use of a multivariate model that relates the attenuation of a spectral feature to a reference coating thickness measurement. Alternative approaches are terahertz pulsed imaging (TPI) and optical coherence tomography (OCT), which are both structural imaging techniques that do not require a multivariate model. Both methods facilitate the direct measurement of the coating thickness by providing depth profiles of the coated tablets during manufacturing. TPI and OCT can be considered as complementary methods where OCT is highly suitable for measuring thin coatings (15 to $100 \mu \mathrm{m}$, highly dependent on the optical properties of the coating material), whereas TPI is particularly powerful for thick coatings ( 40 to $>200 \mu \mathrm{m}$, low dependence on coating material) (Lin et al., 2018, 2017a, 2017b, 2015).

\section{Models}

Predictive models are essential to develop real-time surrogate methods replacing traditional release tests (EMA, 2012). Considering tablet manufacturing, qualitative models are commonly used for identification of input materials and tablets, whilst quantitative models have been published for content, content uniformity, form, water content and dissolution (see Table C.1 for examples). The models can be based on first principles if a simple relationship can be defined between process parameters and the quality attribute of interest, e.g. compaction force as a surrogate for tablet mass (Manley at al., 2019). These models can predict the property of interest in a short time requiring minimal data for calibration and validation. However, in most cases the underlying mechanistic equations are highly complex resulting in long computation times. Moreover, most mechanistic models do not account for small, random variability in process parameters and attributes, i.e. stochastic variation. Therefore, models supporting RTRT are typically data-driven (empirical). Empirical models can be based on off-line data (e.g. raw material attributes, process parameters) and/or real-time process measurements (e.g. tablet hardness, PAT measurements) (EMA, 2012; Zaborenko et al., 2019). Most surrogate methods for batch release of tablets predict quality attributes from at-line or on-line spectral measurements. One exception is the RTRT for tablet dissolution of an AstraZeneca drug product approved by the EMEA (Herkert et al., 2008), which was 
supported by a model based on input material and tablet attributes (accounting for variability in process parameters, see section 6.1).

\subsection{Model Development and Implementation}

All models supporting real-time release are categorised as high impact models, because the resulting predictions are significant indicators of quality (ICH, 2011; Kourti et al., 2014). Guidance for model development and validation are provided by ICH (2011) and EMA (2014). In general, a model development procedure consists of several steps: (1) composition of calibration and validation data set, (2) measurements, (3) data pre-processing, outlier detection and removal, (4) model calibration, (5) validation and (6) lifecycle management.

The selection of calibration and validation data is a critical step ensuring robustness of the release test (EMA, 2014; ICH, 2011): the supporting samples should be representative of the commercial production process and the expected process variability, e.g. API concentration, particle size, material suppliers, equipment and scale. Key considerations of the sampling plan are discussed in Section 5. Therefore, calibration sets are typically generated by varying process parameters and material attributes as part of a DoE within the defined operation ranges (Hetrick et al., 2017).

Spectroscopic data contain noise caused by for example physical scattering effects and fluorescence. which is not related to the predicted CQA(s). Therefore, a wide array of pre-processing techniques can be used to suppress these effects (e.g. standard normal variate transformation, multiplicative scattering correction) and obtain more accurate and precise predictions (Gautam et al., 2015; Rinnan et al., 2009). During this step it is important to identify outliers caused by measurement errors or atypical samples, so they can be removed from the calibration set increasing model accuracy and precision.

As a CQA is mostly described as a function of multiple process parameters and/or spectral attributes, calibration methods are generally using multivariate models such as multiple linear regression (MLR) for DoE applications and PLS regression for spectroscopic applications (Gautam et al., 2015; Wold et al., 2001). Models are typically developed off-line, which is even feasible for on-line RTRT's for CM (Hetrick et al., 2017). During model calibration, it is critical to define statistical limits for spectral and/or process data ensuring validity of the model for every prediction. Every measurement outside these limits is then considered an outlier for which the RTR method is not valid. Recurrent excursions should trigger an investigation as they might indicate problems with the PAT probe or flag the need for a model update. The statistical limits are typically based on score values (e.g. the $99 \%$ confidence region of Hotelling's $T^{2}$ ) and residuals (e.g. distance to model or $Q$ residuals) calculated from the calibration measurements (EMA, 2014). 
351

352

353

354

355

356

357

358

359

360

361

362

363

364

365

366

367

368

369

370

371

372

373

374

General guidance for model validation is provided by the USP, e.g. setting acceptance criteria (USP, 2017) and $\mathrm{ICH}$, e.g. comparison of accuracy of calibration versus prediction, and validation of the model using an external data set, i.e. data set from experiments/batches not used for model building (ICH, 2011, 1995). The EMA (2014) validation requirements for models supporting NIR applications are more detailed covering specificity, linearity and range, accuracy, precision and robustness.

\subsection{Model Lifecycle Management}

Once a RTR test is implemented to support commercial manufacturing, a lifecycle management plan is required as part of the quality management system of the site. This plan should describe a risk-based verification approach for model performance, including both model monitoring and model maintenance. Critical components of the lifecycle management plan are events triggering model updates (e.g. changes in raw materials or equipment), criteria for recalibration and the level of revalidation (Chatterjee, 2019; $I C H, 2011)$. Specific NIR guidance from the FDA (2015) and the EMA (2014) outlines expectations and requirements for model lifecycle management on NIR related applications.

\section{RTRT Development Workflow}

This section presents a systematic and science-based development workflow (Figure 3) for the robust design of an RTRT environment for hard (PAT) and/or soft sensors that enables the determination of a CQA. Details about the different stages and decisions as shown in Figure 3 are discussed below. Note that a certain level of process understanding is required at the start of the workflow but typically additional process understanding is gained during RTRT development.

Stage $1 \mathrm{a} / \mathrm{b}$ and Decision 1: Initially, process and product knowledge need to be gathered and/or generated if not sufficient information is available. PAT methods or soft sensors may need to be developed first to generate process and product understanding. Once sufficient understanding about the processes and product exists, the detailed definition of the attribute of interest should be compiled (stage 2).

Stage 2: The attribute of interest and the overall goal of the RTRT measurement including the physical scale of the attribute, i.e. microscopic (blend homogeneity) vs macroscopic (granule density, granule size/distribution) are defined. This can be summarised in an Analytical Target Profile (Jackson et al., 2019) to prospectively define the requirements of the method. A key element to consider, which can impact the point of testing, is whether the control strategy is intended for monitoring only or real-time feedback control is required (Yu et al., 2014). For example, in batch manufacture, considering the drug product CQA content uniformity, measurement of blend 
homogeneity can be performed at the tablet press feed frame for monitoring, but will need to be performed at the blending stage for end point control. In CM, there is less distinction, but the residence time needs to be considered between the point of testing and the point of control. $A$ detailed definition of the attribute of interest the overall goal of the RTRT measurements is crucial when moving to stage 3 , where the sensor and the type of measurement is selected.

Stage 3: The sensor, location and type of measurement (direct vs indirect measurement of the attribute of interest) are evaluated and defined The decision is predicated on the technical feasibility for direct measurement and the role of the measurement in the context of the control strategy, e.g. tablet weight or compression force can be positioned as an appropriate indirect measurement (i.e. soft sensor) of tablet content if blend homogeneity is determined upstream. The information and knowledge generated in the stages $1-3$ will then be used to perform a risk and cost-benefit analysis (stage 4).

Stage 4: The risk and cost-benefit analysis should consider measurement characteristics, such as selectivity, accuracy and precision, calibration technology transfer, commercialisation, and maintenance. In addition, the effect of the sampling location on the measurement and the process operation needs to be analysed. This is particularly important when using sub-optimal location due to limited availability of existing ports and windows. The process dynamics and intended application should be considered when choosing a point of testing. This risk assessment should also take into account the effect of the sensor on normal process operation and cleanability. Benefits and challenges of the most common points of testing in tablet manufacturing are given in Table 1. In addition, consideration should be given to whether the measured attribute could change downstream from the point of testing (Moore, 2011). For example, given the vicinity of the measurement location to tablet compression, the correlation of API content in final blend in the feed frame with that in tablets has been proved to be robust and reliable. In addition, during early development studies, it was discovered that powder mixing does not end at the outlet of mixer and that the feed frame is also an efficient mixer (Joseph et al., 2011). Thus, the blend homogeneity is best represented at the feed frame, not the mixer. Therefore, many companies use the API content measured at the feed frame to (1) measure real-time API content in the final blend and make real-time accept/reject decision (process disturbance detector) and (2) use such an approach to replace traditional content uniformity release testing. The outcome of the risk and cost-benefit analysis will drive the decision whether RTRT will be realised (decision 2).

Decision 2: Besides the considerations highlighted in Stage 4, this decision needs to consider that product quality needs to be within specification taking into account prediction error as well model diagnostics ensuring the validity of the model at any time. 
Decision 3: The selection of soft sensor versus hard sensor should be made based on the risk assessment and the knowledge developed in the Stages $1-4$. It should also consider the prediction error, required maintenance and validation efforts for a soft or a hard sensor. In the majority of cases, a reference method (Stages R1 - R2) needs to be developed to validate the RTRT method.

Stages R1 - R2: A reference method is typically needed to validate the soft or hard (PAT) sensor. The reference method development may need to consider an additional validation method. More details on reference methods and method validation development can be found in FDA (2015a). The reference method is used in the soft (Stages S1 and S2, Decision S1) or hard sensor development (Stages H1 - H5, Decisions S1 - S3).

Stages S1 - S2, H1 - H3 and Decisions S1, H1 - H2: In the majority of cases a calibration is needed for both soft and hard sensors. The calibration includes the parameter estimation of mechanistic and statistical (multivariate) models that relate the measured signal (PAT or process parameters) to the CQA. Key considerations in the soft and hard sensor development are thus concerning the model development, validation and maintenance, which are discussed in detail in Section 4. The hard sensor development commonly includes more stages and decisions as detailed below.

Decision H3: When choosing between online and inline measurements, there is a trade-off between accuracy versus speed. Is a high sampling frequency critical for monitoring or controlling the attribute of interest? The sampling frequency and measurement duration is part of the sampling plan development, which is discussed below in the Stages H5 and H6.

Stage $\mathbf{H 4 a - b : ~ T h e ~ m e t h o d ~ o f ~ i n t e r f a c i n g ~ t h e ~ m e a s u r e m e n t ~ s y s t e m ~ t o ~ t h e ~ m a n u f a c t u r i n g ~ e q u i p m e n t ~ n e e d s ~}$ to be defined. Effects of the physical and chemical changes of the sample material as well as process and environmental conditions on the measured attribute must be understood to ensure a robust and reliable measurement. The material and process equipment interactions can also impact the window fouling management (Stage $\mathrm{H} 4 \mathrm{c}$ ).

Stage H4c: An understanding of the probability of window fouling, i.e. the build-up of material on or in front of the sensor, needs to be developed. An appropriate procedure (e.g. automated and frequent probe cleaning) should be considered to avoid that window fouling compromises the measurement. The window fouling management will also influence the sampling plan (Stage H5).

Stage H5: Key considerations for developing a robust and reliable sampling plan are discussed in detail in ASTM (2018). In general, the sample size of a PAT measurement should be representative of a unit dose and consider scientific and statistical principles in combination with appropriate risk assessment tools to ensure that the sampling approach is suitable for the application (Lee et al., 2015). This stage includes an analysis of the contribution of a sample to the measurement in terms 
of effective sample size, heterogeneity of the sample material at the micro level, speed of analysis and relative displacement of the sample for a moving process. For example, the effective sample size might be very large if the sample moves quickly compared to the measurement speed. An appropriate measurement cycle time should be determined by considering the overall time-frame and dynamics of the process as well as the effective sample size. Although this section focuses on on/in-line applications of solids, many of the considerations are also relevant for at-line and offline analysis in which a sample is taken from the process and used for analysis.

1. Identify factors affecting the PAT measurement (ruggedness/insensitivity with respect to interferences from other factors). The use of prior knowledge of product and risk assessment in the design of the sampling plan is critical to develop appropriate actions that reduce, mitigate or control these risks. In particular, a risk assessment of sampling errors and its impact on the measurement, reliability of the measurement and the sampling system should be conducted and a mitigation plan should be developed.

2. Understand impact of scale of the manufacturing process (sampling frequency might be higher at small scale compared to full commercial scale, different process dynamics).

3. Understand and consider sample heterogeneity (commercial scale manufacturing may be subject to an increased likelihood of subpopulations which would increase sample-to-sample variations).

4. Estimate the effective sample size (amount of sample which is being investigated). Sensors have a limited field of view and effectively only evaluate a sub-set of the material under investigation. PAT methods will penetrate a sample finite depth and measurements can only be taken at a finite rate.

5. Review the effective samples size in terms of the required level of scrutiny, e.g. blend content uniformity sample size needs to be comparable to the nominal tablet unit dose level. Extremely low dose and/or high potency may need a more rigorous sampling to assess blend and/or dosage unit uniformity.

Stage 5a - c: Once the RTRT sensor is developed, there are several stages that consider the operational procedure. Firstly, in the case of a $\mathrm{CM}$ a residence time distribution needs to be estimated using a digital twin and/or experimental data. The residence time distribution (RTD) may also inform the sampling plan in Stage H5. The RTD is the probability distribution of time that a material (solid or liquid) spend in one or more unit operations (Gao et al, 2012). Secondly, an outlier and maintenance management plan should be developed. This includes the development of a contingency plan for the event of a sampling and system failure as well as human error. This may include parallel monitoring to identify a sampling failure and to ensure the process stays in a state 
of control. Thirdly, the RTRT sensor needs to be integrated in the supervisory control system that manages the storage of the data and real-time control actions (e.g. divert to waste).

Decision 4: As discussed above, a contingency plan for the event of a sampling or system failure can be built into the control strategy for RTRT. The concept of a parallel method can be implemented to confirm the primary (RTRT method) has made the correct decision. As an example, a primary method is used for tablet content using a soft sensor, which has a measurement frequency/periodicity to see high process variance and compliant with sampling requirements for RTRT (Stage H5). A parallel method (spectroscopic measurement) which operates at a much lower frequency is then just used to confirm the soft sensor is still predicting correctly. In case of a deviation between the primary and parallel method, it becomes a risk management issue. If the primary method is still valid, then the manufacturing can be continued but it runs at risk and an investigation needs to be started. The outcome of the investigation could be: i) if the primary method is correct, then the parallel (a low impact model) needs to be updated, or ii) if the primary method is not correct, then the material cannot be released until the method is corrected and revalidated. In regular operation it is necessary to include several parallel low-risk impact methods that can run at a lower frequency and which are designed to verify that the decisions made by the primary methods are correct. These parallel methods do not make the control decision and disagreement between the primary and parallel is captured as a deviation when the process is running.

\section{Industrial Use Cases}

This section provides industrial RTRT examples from AstraZeneca and Eli Lilly.

\subsection{Use Case from AstraZeneca}

At AstraZeneca a real-time process was deployed for the release of a selected commercial product, i.e.an immediate-release tablet manufactured by standard wet granulation and tablet compression in batch mode. The building blocks of the PAT and model-based process monitoring and control strategy ensuring the quality of the final product are outlined in Figure 4, whilst the detailed RTR scheme is summarized in Table 2. This holistic release approach covers the manufacturing process from raw materials at dispensing through to the finished product after compression and coating. By combining in-process monitoring, PAT based controls, and cGMP the intended quality of the finished tablet is assured as well as its compliance with the drug product specification. In essence, this enables release of the manufactured product in realtime by making any testing on the finished product to become redundant postprocessing. Notably, this approach and scheme was the first that achieved regulatory approval of RTR in Europe in 2007 (Herkert 
515

516

517

518

519

520

521

522

523

524

525

526

527

528

529

530

531

532

533

534

535

536

537

538

539

540

541

542

543

544

545

546

547

and Zahn, 2008). Moreover, this preceded the publication of the harmonized guidelines comprising RTRT and its regulatory definition (ICH, 2008).

In detail, the overall strategy is founded on a science- and risk-based combination of standard in-process controls (e.g. tablet hardness), current good manufacturing processes (e.g. material dispensing), and a wide range of PAT and modelling tools, which all support the real-time batch release monitoring and control strategy as described below.

The raw material in every container was identified by measuring diffuse reflectance with an inserted NIR probe. The collected spectra were pre-processed to filter out noise and analysed using a PCA. The resulting PCA scores were then subjected to soft independent modelling by class analogy (SIMCA) to confirm the identity of the material. Tablet identity, content and content uniformity were determined by a real-time automated NIR method in reflectance mode. Sampling was conducted by an automated means that deflected tablets for NIR analysis, where the NIR analyser was either at-line in the manufacturing area or integrated into the tablet compressor. Standard pre-processing techniques and partial least squares regression were applied to predict the quality attributes from the measured spectra, whilst SIMCA was applied for identification. The calibration models for all NIR applications were based on samples generated by a DoE varying API concentration and selected material attributes and process parameters within the established manufacturing ranges. Variations in suppliers for raw materials were also included. The acceptance criteria for content and content uniformity were based on operational curves (i.e. probability of acceptance versus proportion of units with content in the range of 85 - 115\%) accounting for the large sample sizes (Sandell et al., 2006). The model validation approach addressed selectivity, linearity and range, accuracy ( 2 external validation sets), precision and robustness.

For each batch, the dissolution was predicted by a multiple regression model based on (1) mechanistic understanding of the effect of key materials and processing parameters on tablet product dissolution properties and (2) a complementary experimental design varying selected process parameters and attributes simultaneously at laboratory scale. In the DoE, input material and tablet core attributes and process conditions were provoked far outside normal operational ranges. The statistically significant design factors, i.e. two input material and one tablet attribute, confirmed mechanistic understanding and were therefore retained in the model containing linear, interaction and quadratic terms. The regression model was evaluated based on mechanistic understanding of in-vitro dissolution, statistic diagnostics (e.g. lack of fit, residuals) and further verification with two external data sets generated from more than ten full scale batches. Note that the model is only valid when all process parameters and attributes are within the established boundary conditions, e.g. manufacturing process ranges and specification acceptance criteria of the API. The prediction model can also be used to finetune the output of the manufacturing 
process in a feed forward control way, by adjusting any of the modelled attributes, i.e. advanced process control.

Note that the real-time release control strategy was complemented by traditional release testing for API purity, granule moisture content and tablet appearance, whilst the microbial quality was controlled by cGMP (Table 2).

\subsection{Use Case from Eli Lilly}

A feed frame PAT tool was developed and deployed at Lilly to enable real-time determination of API concentration in the final blend for a CM process of a drug product. The PAT tools here refer to a spectroscopic tool capable of measuring API concentration in the powder format. The main driver to choose the feed frame as the sampling location is its representativeness of content uniformity on individual solid dosage forms. For late-phased assets, an off-line feed frame table was developed to collect calibration spectra in order to mimic the dynamic powder movement inside feed frame without running the entire CM line to save on material and resources. The subsequently developed PLS model is then used to serve as a real-time accept/reject tool to assure the product quality. Given the unique location of feed frame and its representativeness for content uniformity, such a tool can be naturally upgraded to replace routine content uniformity release testing on tablets. For early-phase assets, a lean modelling routine (Shi et al., 2019) was developed to determine real-time API concentration at the same location without using a full quantitative DoE-based calibration data set. The value added of such a lean tool was to provide process understanding in early R\&D, perform real-time accept/reject and assure the product quality on the clinical trial (CT) batches. No RTRT on content uniformity has been investigated for releasing those clinical trial materials.

In general, an RTRT approach for content uniformity must statistically ensure compliance with USP <905> requirements (USP, 2011). This was addressed by developing a process control strategy that ensures collection of tablets within pre-defined tablet weight limits and API concentration (mixing homogeneity) limits in the feed frame (Table 3). This approach triggers automated removal of suspect tablets outside these limits via the tablet press discharge chute. In order to meet the USP content uniformity pharmacopeia requirements, a high percentage of the dosage units must be within $85 \%$ to $115 \%$ of label claim (Bergum, 2015). Pre-defined limits can be adjusted to ensure dosage units are within $85 \%$ to $115 \%$ of label claim providing assurance that the USP Content Uniformity requirements are met. This RTRT case has been accepted as part of a control strategy in multiple markets. The two specific elements of the control strategy contributing to this assertion are the following:

1. The tablet press control strategy makes product collection decisions on $100 \%$ of the tablets based on the individual tablet main compression force value. The individual main compression 
force limits will be set to collect tablets within the desired tablet weight control limits (Manley et al., 2019).

2. The feed frame NIR concentration control strategy ensures collection of tablets when the predicted API concentration in the tablet press feed frame is within predefined limits.

The example in Figure 5 has individual tablet weight limits set at $95-105 \%$ of target ( $y$-axis) and drug substance concentration limits of $90-110 \%$ (x-axis). The black contours are the resulting label claim and are a function of the tablet percent of target weight and the percent of theoretical concentration.

\section{Discussion and Future Perspective}

The value of RTRT for pharmaceutical manufacturing was demonstrated in this paper by the review of existing literature as well as by introducing new case studies. It was shown that tests for identity, assay and content uniformity are well established, whilst more challenging applications supporting tablet dissolution (e.g. dissolution models, tablet porosity tests) are also being tackled. These RTRT's achieve high sampling frequencies supporting the evaluation of both intra- and inter batch variability, hence increasing quality assurance. The high speed of these tests improves manufacturing efficiency by accelerating batch release and by increasing process flexibility. The latter is achieved by optimising process operations based on real-time quality insights, e.g. fine tune process parameters based on models underlying RTRT, divert non-confirming material to waste based on real-time quality reading instead of discarding entire batch after completion. Although accurate quantifications for low dose formulations or moving materials remain challenging, the wide range of published PAT applications indicates that RTRT can be implemented routinely to monitor all CQAs. An RTR approach reduces production times and costs significantly by eliminating the need for end-product testing partially or completely but requires more maintenance for supporting PAT equipment and models. This requires a capability shift at pharmaceutical manufacturing plants increasing the number of PAT and modelling specialists. Once fully established, routine RTRT can drive performance-based control strategies as introduced in ICH Q12.

Remaining hurdles with regards to further establishing and growing the application of RTRT in industrial practice considering technical, logistical, management and regulatory challenges are described below.

\subsection{Technical Aspects}

On the technical side, there is a continued need for highly sensitive and fast analytical sensors that are capable of sampling a significant and well-defined volume of powder or dosage form to make reliable non-destructive measurements of API content in low dose tablets as well as mechanical and structural properties. In particular, granule and tablet porosity are CQAs which have a direct impact on the in vitro dissolution kinetics of tablets (Desai et al., 2016; Markl et al., 2018b; Markl and Zeitler, 2017; Quodbach and Kleinebudde, 2015). These structural properties are primarily introduced by the manufacturing and 
614

615

616

617

618

619

620

621

622

623

624

625

626

627

628

629

630

631

632

633

634

635

636

637

638

639

640

641

642

643

644

hence minor variations in the process and environmental conditions can cause a significant change in granule and tablet porosity (Markl et al., 2018c, 2017a). Although progress has been made in developing new methods for measuring tablet porosity (Markl et al., 2018b, Svensson et al., 2007), it will take some time to bring these technologies from a research environment into an industrial setting.

\subsection{Operational Aspects}

Key challenges are to maintain the accuracy and precision of the sensor equipment during process conditions and over long periods (years). Small changes in hardware can invalidate the registered calibration model and hence require an update. The risk from this aspect is reduced by selecting suitably robust and well-established methods with a proven track record in quality control.

From a logistical and management perspective, the implementation of RTRT requires more PAT and modelling experts to be available at manufacturing sites to support the equipment and models that are at the core of the present approach. This causes particular difficulties when transferring such a method from one manufacturing site to another if manufacturing is shifted to a different facility or if additional processing sites are required due to supply chain considerations.

Furthermore, the models typically require frequent updates as a result of additional variability in the raw material, that is not easily foreseen during development, but is only emerging with time when suppliers change or simply due to fluctuations in the process or starting materials. Additional sources of variability are instrument instability (e.g. drift over time), adaptations to process equipment or the process itself. While the frequency of model maintenance can be greatly reduced by carefully optimising the model robustness during development, it cannot be avoided altogether. One strategy to overcome this challenge could be the automation of model calibration and validation to reduce the resources required from expert personnel. This approach would benefit from support from the regulatory authorities by facilitating suitable post-approval changes for models.

One of the consequences following the implementation of RTRT is that the higher sampling frequency in quality testing increases the amount of data that needs to be processed, stored and curated. Suitable solutions need to be developed and refined that accommodate all requirements with regards to data integrity, traceability of quality data, data storage and associated logistics.

From a risk management perspective, a contingency plan is required to mitigate against the failure of the sensor systems that are in place to ensure RTRT. A potential fallback position is to revert to traditional quality testing as a contingency, but implementing parallel method into the RTRT framework may be a more desirable option. 


\subsection{Regulatory Aspects}

Since the introduction of PAT in the pharmaceutical industry, the pharmaceutical companies, together with the three central regulatory bodies, have devoted a tremendous amount of resource to improve the quality of medicines through PAT and RTRT. Whether and how far this transformation of pharmaceutical processing can advance in the coming decade will depend mainly on overcoming the hurdles discussed in this section. The rewards from adopting this technology both in terms of reduced waste, and in turn reduced manufacturing costs, as well as the improved quality of medicines on the market, are reason enough to enthusiastically continue to push for broader adoption of this approach in the pharmaceutical industry.

Currently, the level of detail subjected to regulatory changes for spectroscopic applications are often higher in comparison with traditional analytical testing procedures. Most common, low-risk model updates, for example, require prior approval which can be prohibitive for RTRT applications as processing would need to be paused until regulatory approval was obtained. Given that agile model maintenance is critical for real-time in-process control and release applications, the general principle should be that method and model maintenance of PAT methods are handled within a company's internal pharmaceutical quality system. The European Federation of Pharmaceutical Industries and Associations (EFPIA) proposed a more flexible approach in June 2018. Currently, the regulatory framework is still developing actively alongside the industrial adaptation of RTRT. Given that the EMA is revising its NIR addendum to reduce the variation reporting category, it appears not unreasonable to assume that the regulatory expectation on model lifecycle management is gradually merging with the trend of established conditions as outlined in ICH Q12. Such a development would be highly desirable from a PAT practitioners' standpoint since it would ultimately give the autonomy of model lifecycle management to the local manufacturing site as soon as the established conditions are satisfied.

Lack of harmonisation with regards to regulatory expectation presents a pivotal challenge to the more widespread adoption of RTRT moving forward. The FDA, EMA and PMDA have pioneered and lead the approval of PAT and RTRT for medicine manufacturing and have developed suitable mechanisms to support the sustained use of PAT and RTRT, such as the EMA's notification tool (do and then tell) compared to the traditional approval mechanism (tell, approve and then do), hence preventing extensive manufacturing pauses awaiting regulatory approval for a model update. For the regulatory authorities in the rest of the world, the level of engagement and degree of openness is less clear. Further harmonisation and wide adoption of the regulations developed by the agencies mentioned above would be highly beneficial also for the remainder geographic regions. In this context, it is essential not merely to update the regulations to allow the use of RTRT, but also to provide the necessary training for the reviewers and inspectors to streamline the common technical document (CTD) review and on-site inspection. 
679 Besides the use of well-established simple sensors to capture physical properties such as temperature, 680 humidity, pressure and spatial dimensions there is a need for more advanced process sensors to assess 681 CQAs specific to pharmaceutical products. Optical sensors in general, and spectroscopy sensors, in 682 particular, have been at the core of RTRT developments in the pharmaceutical industry. In that regard, 683 there is typically a preference to opt for a conservative choice from the available sensor technologies 684 (such as NIR, Raman and light scattering methods) to mitigate the risk of sensor failure or unreliable 685 readings. This is a sensible and pragmatic choice but one that can ultimately result in missing out on 686 opportunities for better quality testing through emerging, more advanced, technologies. In the authors' 687 opinion, the present regulatory context is unfavourable for the adoption of new sensor technologies as 688 part of the lifecycle management of a product manufactured under RTRT. It is therefore essential to 689 maintain an open mind in implementing novel approaches in terms of sensor technology platform, data 690 processing and model building approaches during the development of new products instead of merely 691 adopting 'tried-and-tested' methods from previous products when developing RTRT solutions in existing 692 process facilities.

693

694

695

696

697

698

699

700

701

702

703

704

705

706

707

708

709

Regulatory agencies encourage the pharmaceutical industry to seek their scientific advice at an early stage of the development or adoption of new technologies. Pathways for the industry to interact with the regulatory agencies can include the request of written guidance as a response to a set of questions or the arrangement of face-to-face meetings between the two parties. For example, FDA's Emerging Technology Program can be used to request either a pre-submission or a regulatory submission interaction with the Emerging Technology Team (ETT). This interaction can be utilised to discuss a novel or impactful product technology (e.g. new dosage form), manufacturing process (e.g. novel manufacturing process) or control strategy technology (e.g. PAT enabled IPC and RTRT). The ETT typically responds to questions from the industry in the form of a written guidance and/or a face-to-face meeting. The interaction with the ETT can also include a Pre-Operational Visit (POV), where FDA reviewers visit company facilities (e.g. commercial manufacturing facility) to discuss every aspect of the common technical document (CTD) including PAT enabled IPC and RTRT. Such interactions do not only allow the industry to increase their understanding of the regulatory agency's expectations, but also facilitate the learning of a regulatory agency about the new technology.

\section{ACKNOWLEDGEMENTS}

The authors would like to thank the working group of the International Society for Pharmaceutical Engineering (ISPE) UK PAT Affiliate for their valuable discussions. 


\section{APPENDICES}

\section{APPENDIX A: Definitions}

Pharmaceutical control strategy

A pharmaceutical control strategy describes the methodology that is put in place to ensure a process stays in a state of control throughout the operation and thus ensuring product quality $(\mathrm{ICH}$, 2007).

Analysis method

A method is considered as the whole system which could include the measurement instrument, data processing steps, quantitative prediction of a CQA and the control decision of the Critical Process Parameter (CPP) for Level 3. Often methods have a staged life cycle: Stage one collects data to build models, stage two adds the realtime prediction, and stage three is the on-going maintenance of the method/model on a performance basis. As a result, these methods do not only require qualified equipment but also that the methods must be validated according to the guidelines set out in 'ICH Q2 (R1) Validation of Analytical Procedures: Text and Methodology' (ICH, 1995). An equipment is considered qualified if it is described and maintained under the pharmaceutical quality system (PQS), which means that there will be a pre-approved protocol, execution and qualification report including protocols for use, maintenance (user level) and periodicity of requalification.

Primary method Methods that are used to make quality-based control decisions (Vogt and Kord, 2011).

Parallel method

The concept of a parallel method can be implemented to confirm a primary (RTRT method) has made the correct decision. Parallel methods do not make the control decision.

Quality risk management A systemic process for the assessment, control, communication and review of risks to the quality of the drug (medicinal) product across the product lifecycle (ICH Q9, 2015).

Critical quality attribute

"A CQA is a physical, chemical, biological, or microbiological property or characteristic that should be within an appropriate 
limit, range, or distribution to ensure the desired product quality."

(ICH, 2009)

712

713

714

715

716

717

718

719

720

721

722

723

724

725

726

727

728

729

730

731

732

733

734

735

736

737

738

739

740

\section{APPENDIX B: Pharmaceutical Control Strategy}

\section{B.1 Level 3 Control Strategy - Recipe Control}

For processes running under recipe control, the control strategy ensures that the process stays within an acceptable range (delta) for all process control parameters, followed by using 'end of line testing' to ensure the product has reached the desired quality. Although this is the most straightforward approach, there is little forward flexibility to operate the process. This type of control strategy does not require or benefit from an approved multivariate process space (Design Space, or DSp) and relies on 'end of line testing' to confirm product quality. Such testing needs to be carried out at a statistically representative frequency or spatial location to ensure any perturbation in the process is caught. In $\mathrm{CM}$, the residence time distributions of the process streams need to be well established as a minimum process understanding at least at the level of the unit operations.

\section{B.2 Level 2 Control Strategy - Pharmaceutical Control}

A step up in complexity is the Level 2 Control Strategy, often referred to as pharmaceutical control. Here all critical unit operations have an approved DSp while non-critical unit operations remain under recipe control. The approved DSp is described by the relationship between critical process parameters (CPP), which represent the multivariate operating space that ensures the final product outputs remain within acceptable quality limits. Action limits on the CPP are then defined and set to ensure that the process remains within the approved DSp. In addition, chemical, physical, biological and microbiological attributes of inputs, intermediates and outputs are monitored to demonstrate state of control.

\section{B.3 Level 1 Control Strategy - Engineering Control}

The highest level of control strategy takes the approved DSp and prediction models from Level 2 and also applies automated controllers. As a result, model predictive control is achieved for the process, and with that, it is possible to actively steer the process to different conditions within the DSp to achieve and maintain the desired product quality. It is worth noting that in order to achieve Level 1 control, typically process models are required. Given the reduction in uncertainty in the predictions that can be achieved through the before-mentioned models, it can be justified to no longer require parallel methods in Level 1 control.

It is worth noting that a continued or on-going process verification plan always needs to be in place to capture the performance of the DSp, the primary and parallel methods (to trigger method maintenance) 
741 but also to verify the initial criticality assessment in order to ensure non-critical process steps do not

742 transition to critical during the life-cycle of the product.

\section{APPENDIX C: Summary of Literature}

744 The literature of RTRT applications is summarised in Table C.1.

\section{REFERENCES}

746

747

Ahlneck, C., Alderborn, G., 1989. Moisture adsorption and tabletting. I. Effect on volume reduction properties and tablet strength for some crystalline materials. Int J Pharmaceut 54, 131-141. https://doi.org/10.1016/0378-5173(89)90332-3

Almaya, A., Belder, L., Meyer, R., Nagapudi, K., Lin, H., Leavesley, I., Jayanth, J., Bajwa, G., DiNunzio, J., Tantuccio, A., Blackwood, D., Abebe, A. (2017). Control strategies for drug product continuous direct compression - state of control, product collection strategies, and startup/shutdown operations for the production of clinical trial materials and commercial products. J Pharm Sci 106(4), 930-943. https://dx.doi.org/10.1016/j.xphs.2016.12.014

ASTM, 2018. Standard Guide on Sampling for Process Analytical Technology. ASTM International.

Austin, J., Gupta, A., Mcdonnell, R., Reklaitis, G.V., Harris, M.T., 2013. The Use of Near-Infrared and Microwave Resonance Sensing to Monitor a Continuous Roller Compaction Process. J Pharm Sci 102, 1895 1904. https://doi.org/10.1002/jps.23536

Beer, D.T., Burggraeve, A., Fonteyne, M., Saerens, L., Remon, J., Vervaet, C., 2011. Near infrared and Raman spectroscopy for the in-process monitoring of pharmaceutical production processes. Int J Pharm 417, 32 47. https://doi.org/10.1016/j.ijpharm.2010.12.012

Bergum, J., 2015. Tolerance interval alternative to ASTM E2709/E2810 methodology to provide assurance of passing the USP uniformity of dosage unit (UDU) test< 905>. Pharm Eng 68-79.

Berntsson, O., Danielsson, L.-G., Lagerholm, B., Folestad, S., 2002. Quantitative in-line monitoring of powder blending by near infrared reflection spectroscopy. Powder Technol 123, 185-193. https://doi.org/10.1016/s0032-5910(01)00456-9

Blanco, M., Alcalá, M., González, J.M., Torras, E., 2006. A process analytical technology approach based 
774

775

776

777

778

779

780

781

782

783

784

785

786

787

788

789

790

791

792

793

794

795

796

797

798

799

800

801

802

803

804

805

806

807

on near infrared spectroscopy: Tablet hardness, content uniformity, and dissolution test measurements of intact tablets. J Pharm Sci 95, 2137 2144. https://doi.org/10.1002/jps.20653

Burggraeve, A., Kerkhof, V.T., Hellings, M., Remon, J.P., Vervaet, C., Beer, D.T., 2010. Evaluation of in-line spatial filter velocimetry as PAT monitoring tool for particle growth during fluid bed granulation. Eur J Pharm Biopharm 76, 138-146. https://doi.org/10.1016/j.ejpb.2010.06.001

Buschmüller, C., Wiedey, W., Döscher, C., Dressler, J., Breitkreutz, J., 2008. In-line monitoring of granule moisture in fluidized-bed dryers using microwave resonance technology. Eur J Pharm Biopharm 69, 380387. https://doi.org/10.1016/j.ejpb.2007.09.014

Chablani, L., Taylor, M.K., Mehrotra, A., Rameas, P., Stagner, W.C., 2011. Inline Real-Time Near-Infrared Granule Moisture Measurements of a Continuous Granulation-Drying-Milling Process. AAPS

PharmSciTech 12, 1050 1055. https://doi.org/10.1208/s12249-011-9669-z

Chatterjee, S., 2019. Implementing models in pharmaceutical manufacturing: FDA perspective.

Crouter, A., Briens, L., 2019. Methods to Assess Mixing of Pharmaceutical Powders. AAPS PharmSciTech 20, 84. https://doi.org/10.1208/s12249-018-1286-7

Desai, P., Liew, C., Heng, P., 2016. Review of Disintegrants and the Disintegration Phenomena. J Pharm Sci 105, 2545 2555. https://doi.org/10.1016/j.xphs.2015.12.019

Donoso, M., Kildsig, D., Ghaly, E.S., 2003. Prediction of Tablet Hardness and Porosity Using Near-Infrared Diffuse Reflectance Spectroscopy as a Nondestructive Method. Pharm Dev Technol 8, 357-366. https://doi.org/10.1081/pdt-120024689

EMA, 2014. Guideline on the use of near infrared spectroscopy by the pharmaceutical industry and the data requirements for new submissions and variations (EMEA/CHMP/CVMP/QWP/17760/2009 Rev2).

EMA, 2012. Guideline on Real Time Release Testing (formerly Guideline on Parametric Release) (EMA/CHMP/QWP/811210/2009-Rev1) .

FDA, 2015a. Development and Submission of Near Infrared Analytical Procedures: Guidance for 
808

809

810

811

812

813

814

815

816

817

818

819

820

821

822

823

824

825

826

827

828

829

830

831

832

833

834

835

836

837

838

839

840

841

Industry.

FDA, 2015b. Analytical Procedures and Methods Validation for Drugs and Biologics: Guidance for Industry.

Fonteyne, M., Soares, S., Vercruysse, J., Peeters, E., Burggraeve, A., Vervaet, C., Remon, J., Sandler, N., Beer, T., 2012a. Prediction of quality attributes of continuously produced granules using complementary pat tools. Eur J Pharm Biopharm 82, 429 436. https://doi.org/10.1016/j.ejpb.2012.07.017

Fonteyne, M., Vercruysse, J., Díaz, D., Gildemyn, D., Vervaet, C., Remon, J., Beer, T., 2012b. Real-time assessment of critical quality attributes of a continuous granulation process. Pharm Dev Technol 18, 85 97. https://doi.org/10.3109/10837450.2011.627869

Fonteyne, M., Vercruysse, J., Leersnyder, F., Snick, B., Vervaet, C., Remon, J., Beer, T., 2015. Process Analytical Technology for continuous manufacturing of solid-dosage forms. Trac Trends Anal Chem 67, 159 166. https://doi.org/10.1016/j.trac.2015.01.011

Gabbott, I.P., Husban, F., Reynolds, G.K., 2016. The combined effect of wet granulation process parameters and dried granule moisture content on tablet quality attributes. Eur J Pharm Biopharm 106, 70 78. https://doi.org/10.1016/j.ejpb.2016.03.022

Gao, Y., Muzzio, F. J., lerapetritou, M. G., 2012. A review of the Residence Time Distribution (RTD) applications in solid unit operations, Powder Technol 228, 416-423.

https://doi.org/10.1016/j.powtec.2012.05.060

Gautam, R., Vanga, S., Ariese, F., Umapathy, S., 2015. Review of multidimensional data processing approaches for Raman and infrared spectroscopy. Epj Techniques Instrum 2, 8. https://doi.org/10.1140/epjti/s40485-015-0018-6

Goodwin, D.J., van den Ban, S., Denham, M., Barylski, I., 2018. Real time release testing of tablet content and content uniformity. Int J Pharmaceut 537, 183 192. https://doi.org/10.1016/j.ijpharm.2017.12.011

Gupta, A., Peck, G.E., Miller, R.W., Morris, K.R., 2005. Real-time near-infrared monitoring of content uniformity, moisture content, compact density, tensile strength, and young's modulus of roller compacted powder blends. J Pharm Sci 94, 1589 1597. https://doi.org/10.1002/jps.20375 
842

843

844

845

846

847

848

849

850

851

852

853

854

855

856

857

858

859

860

861

862

863

864

865

866

867

868

869

870

871

872

873

874

875

Harms, Z.D., Shi, Z., Kulkarni, R.A., Myers, D.P., 2019. Characterization of Near-Infrared and Raman Spectroscopy for In-Line Monitoring of a Low-Drug Load Formulation in a Continuous Manufacturing

Process. Anal Chem 91, 8045 8053. https://doi.org/10.1021/acs.analchem.8b05002

Hartung, A., Knoell, M., Schmidt, U., Langguth, P., 2010. Role of continuous moisture profile monitoring by inline NIR spectroscopy during fluid bed granulation of an Enalapril formulation. Drug Dev Ind Pharm 37, 274-280. https://doi.org/10.3109/03639045.2010.509725

Hausman, D.S., Cambron, T.R., Sakr, A., 2005. Application of on-line Raman spectroscopy for characterizing relationships between drug hydration state and tablet physical stability. Int J Pharm 299, 19-33. https://doi.org/10.1016/j.ijpharm.2005.03.005

Herkert, T., Zahn, M., 2008. Paving the Way to Regulatory Flexibility: The First Approval of Real-Time Release in Europe. RAJ - PHARMA 19, 155-159.

Hernandez, E., Pawar, P., Keyvan, G., Wang, Y., Velez, N., Callegari, G., Cuitino, A., Michniak-Kohn, B., Muzzio, F.J., Romañach, R.J., 2016. Prediction of dissolution profiles by non-destructive near infrared spectroscopy in tablets subjected to different levels of strain. J Pharm Biomed 117, 568-576.

https://doi.org/10.1016/j.jpba.2015.10.012

Hetrick, E.M., Shi, Z., Barnes, L.E., Garrett, A.W., Rupard, R.G., Kramer, T.T., Cooper, T.M., Myers, D.P., Castle, B.C., 2017. Development of Near Infrared Spectroscopy-based Process Monitoring Methodology for Pharmaceutical Continuous Manufacturing Using an Offline Calibration Approach. Anal Chem 89, 9175 9183. https://doi.org/10.1021/acs.analchem.7b01907

ICH, 2011. ICH Quality Implementation Working Group Points to Consider (R2), ICH-endorsed guide for ICT Q8/Q9/Q10 implementation.

ICH, 2009. Q8(R2) Pharmaceutical Development (step 5).

ICH, 2008. Q8 (R1) Pharmaceutical Development (step 4).

$\mathrm{ICH}, 2007$. ICH guideline Q10 on pharmaceutical quality system. 
ICH, 1995. ICH Q2 (R1) Validation of analytical procedures: text and methodology.

878

879

880

881

882

883

884

885

886

887

888

889

890

891

892

893

894

895

896

897

898

899

900

901

902

903

904

905

906

907

908

909

Jackson, P., Borman, P., Campa, C., Chatfield, M., Godfrey, M., Hamilton, P., Hoyer, W., Norelli, F., Orr, R., Schofield, T., 2019. Using the Analytical Target Profile to Drive the Analytical Method Lifecycle. Anal Chem 91, 2577-2585. https://doi.org/10.1021/acs.analchem.8b04596

Johansson J, Sparén, A., Wikström, H., Tajarobi, P., Lundin, P., Långberg, A., Sebesta, M., Lewander Xu, M. Optical Porosimetry by Gas in Scattering Media Absorption Spectroscopy (GASMAS) Applied to Roller Compaction Ribbons,Int. J. Pharm. 2020.

Järvinen, K., Hoehe, W., Järvinen, M., Poutiainen, S., Juuti, M., Borchert, S., 2013. In-line monitoring of the drug content of powder mixtures and tablets by near-infrared spectroscopy during the continuous direct compression tableting process. Eur J Pharm Sci 48, 680688.

https://doi.org/10.1016/j.ejps.2012.12.032

Johansson, J., Clayboum, C., Folestad, S., Raman spectroscopy: a strategic tool in the PAT toolbox. Emerging biomedical and pharmaceutical applications of Raman spectroscopy, 2010, Springer, London.

Joseph, K.I., Blackwood, D., MacDonald, B., Polizzi, M., Moore, F., Hancock, B., 2011. Rational Design and Scale-Up of Batch Lubrication Process for Immediate Release Formulations.

Karande, A.D., Heng, P., Liew, C., 2010. In-line quantification of micronized drug and excipients in tablets by near infrared (NIR) spectroscopy: Real time monitoring of tabletting process. Int J Pharm 396, 6374. https://doi.org/10.1016/j.ijpharm.2010.06.011

Khorasani, M., Amigo, J.M., Bertelsen, P., Sun, C.C., Rantanen, J., 2016. Process optimization of dry granulation based tableting line: Extracting physical material characteristics from granules, ribbons and tablets using near-IR (NIR) spectroscopic measurement. Powder Technol 300, 120125. https://doi.org/10.1016/j.powtec.2016.03.004

Kleinebudde, P., 2004. Roll compaction/dry granulation: pharmaceutical applications. Eur J Pharm Biopharm 58, 317-326. https://doi.org/10.1016/j.ejpb.2004.04.014

Kourti, T., Lepore, J., Liesum, L., Nasr, M., Chatterjee, S., Moore, C., Korakianiti, E., 2014. Scientific and Regulatory Considerations for Implementing Mathematical Models in the Quality by Design (QbD) 
910

911

912

913

914

915

916

917

918

919

920

921

922

923

924

925

926

927

928

929

930

931

932

933

934

935

936

937

938

939

940

941

942

943

Framework. Pharm Eng 34, 1-21.

Kumar, V., Taylor, M.K., Mehrotra, A., Stagner, W.C., 2013. Real-Time Particle Size Analysis Using Focused Beam Reflectance Measurement as a Process Analytical Technology Tool for a Continuous Granulation-Drying-Milling Process. AAPS PharmSciTech 14, 523 530. https://doi.org/10.1208/s12249013-9934-4

Laske, S., Paudel, A., Scheibelhofer, O., Sacher, S., Hoermann, T., Khinast, J., Kelly, A., Rantannen, J., Korhonen, O., Stauffer, F., Leersnyder, F., Beer, T., Mantanus, J., Chavez, P.-F., Thoorens, B., Ghiotti, P., Schubert, M., Tajarobi, P., Haeffler, G., Lakio, S., Fransson, M., Sparen, A., Abrahmsén-Alami, S., Folestad, S., Funke, A., Backx, I., Kavsek, B., Kjell, F., Michaelis, M., Page, T., Palmer, J., Schaepman, A., Sekulic, S., Hammond, S., Braun, B., Colegrove, B., 2017. A Review of PAT Strategies in Secondary Solid Oral Dosage Manufacturing of Small Molecules. J Pharm Sci 106, 667712.

https://doi.org/10.1016/j.xphs.2016.11.011

Lee, S.L., O'Connor, T.F., Yang, X., Cruz, C.N., Chatterjee, S., Madurawe, R.D., Moore, C.M., Yu, L.X., Woodcock, J., 2015. Modernizing Pharmaceutical Manufacturing: from Batch to Continuous Production. J Pharm Innov 10, 191 199. https://doi.org/10.1007/s12247-015-9215-8

Lin, H., Dong, Y., Markl, D., Williams, B.M., Zheng, Y., Shen, Y., Zeitler, A.J., 2017a. Measurement of the Intertablet Coating Uniformity of a Pharmaceutical Pan Coating Process With Combined Terahertz and Optical Coherence Tomography In-Line Sensing. J Pharm Sci 106, 10751084.

https://doi.org/10.1016/j.xphs.2016.12.012

Lin, H., Dong, Y., Markl, D., Zhang, Z., Shen, Y., Zeitler, A.J., 2017b. Pharmaceutical Film Coating Catalog for Spectral Domain Optical Coherence Tomography. J Pharm Sci 106, 31713176.

https://doi.org/10.1016/j.xphs.2017.05.032

Lin, H., Dong, Y., Shen, Y., Zeitler, A.J., 2015. Quantifying Pharmaceutical Film Coating with Optical Coherence Tomography and Terahertz Pulsed Imaging: An Evaluation. J Pharm Sci 104, 33773385. https://doi.org/10.1002/jps.24535

Lin, H., Zhang, Z., Markl, D., Zeitler, J., Shen, Y., 2018. A Review of the Applications of OCT for Analysing Pharmaceutical Film Coatings. Appl Sci 8, 2700. https://doi.org/10.3390/app8122700 
944

945

946

947

948

949

950

951

952

953

954

955

956

957

958

959

960

961

962

963

964

965

966

967

968

969

970

971

972

973

974

975

976

977
Lourenço, V., Herdling, T., Reich, G., Menezes, J.C., Lochmann, D., 2011. Combining microwave resonance technology to multivariate data analysis as a novel PAT tool to improve process understanding in fluid bed granulation. Eur J Pharm Biopharm 78, 513521.

https://doi.org/10.1016/j.ejpb.2011.02.008

Luukkonen, P., Fransson, M., Björn, I., Hautala, J., Lagerholm, B., Folestad, S., 2008. Real-Time Assessment of Granule and Tablet Properties Using In-line Data From a High-shear Granulation Process. J Pharm Sci 97, 950-959. https://doi.org/10.1002/jps.20998

Manley, L., Hilden, J., Valero, P., Kramer, T., 2019. Tablet Compression Force as a Process Analytical Technology (PAT): 100\% Inspection and Control of Tablet Weight Uniformity. J Pharm Sci 108, 485 493. https://doi.org/10.1016/j.xphs.2018.07.004

Manley, L., Shi, Z., 2018. Characterizing drug product continuous manufacturing residence time distributions of major/minor excipient step changes using near infrared spectroscopy and process parameters. Int J Pharm 551, 6066.

Markl, D., Bawuah, P., Ridgway, C., van den Ban, S., Goodwin, D.J., Ketolainen, J., Gane, P., Peiponen, K.E., Zeitler, A.J., 2018a. Fast and non-destructive pore structure analysis using terahertz time-domain spectroscopy. Int J Pharm 537, 102 110. https://doi.org/10.1016/j.ijpharm.2017.12.029

Markl, D., Hannesschläger, G., Sacher, S., Leitner, M., Buchsbaum, A., Pescod, R., Baele, T., Khinast, J.G., 2015. In-Line Monitoring of a Pharmaceutical Pan Coating Process by Optical Coherence Tomography. J Pharm Sci 104, 2531 2540. https://doi.org/10.1002/jps.24531

Markl, D., Sauerwein, J., Goodwin, D.J., van den Ban, S., Zeitler, A.J., 2017a. Non-destructive Determination of Disintegration Time and Dissolution in Immediate Release Tablets by Terahertz Transmission Measurements. Pharm Res 34, 1012 1022. https://doi.org/10.1007/s11095-017-2108-4

Markl, D., Strobel, A., Schlossnikl, R., Bøtker, J., Bawuah, P., Ridgway, C., Rantanen, J., Rades, T., Gane, P., Peiponen, K.-E., Zeitler, A.J., 2018b. Characterisation of pore structures of pharmaceutical tablets: A review. Int J Pharm 538, 188 214. https://doi.org/10.1016/j.ijpharm.2018.01.017 
978

979

980

981

982

983

984

985

986

987

988

989

990

991

992

993

994

995

996

997

998

999

1000

1001

1002

1003

1004

1005

1006

1007

1008

1009

1010

1011

Markl, D., Wang, P., Ridgway, C., Karttunen, A.-P., Bawuah, P., Ketolainen, J., Gane, P., Peiponen, K.-E., Zeitler, A.J., 2018c. Resolving the rapid water absorption of porous functionalised calcium carbonate powder compacts by terahertz pulsed imaging. Chem Eng Res Des 132, 10821090.

https://doi.org/10.1016/j.cherd.2017.12.048

Markl, D., Wang, P., Ridgway, C., Karttunen, A.-P., Chakraborty, M., Bawuah, P., Pääkkönen, P., Gane, P., Ketolainen, J., Peiponen, K.-E., Zeitler, A.J., 2017b. Characterization of the Pore Structure of Functionalized Calcium Carbonate Tablets by Terahertz Time-Domain Spectroscopy and X-Ray Computed Microtomography. J Pharm Sci 106, 1586 1595. https://doi.org/10.1016/j.xphs.2017.02.028

Markl, D., Zeitler, A.J., 2017. A Review of Disintegration Mechanisms and Measurement Techniques. Pharm Res 34, 890 917. https://doi.org/10.1007/s11095-017-2129-z

Moore, C., 2011. Regulatory perspectives on real time release testing.

Müller, J., Brock, D., Knop, K., Zeitler, A.J., Kleinebudde, P., 2012. Prediction of dissolution time and coating thickness of sustained release formulations using Raman spectroscopy and terahertz pulsed imaging. Eur J Pharm Biopharm 80, 690-697. https://doi.org/10.1016/j.ejpb.2011.12.003

Nagy, B., Farkas, A., Borbás, E., Vass, P., Nagy, Z., Marosi, G., 2018. Raman Spectroscopy for Process Analytical Technologies of Pharmaceutical Secondary Manufacturing. AAPS PharmSciTech 20, 1. https://doi.org/10.1208/s12249-018-1201-2

Nagy, B., Petra, D., Galata, D., Démuth, B., Borbás, E., Marosi, G., Nagy, Z., Farkas, A., 2019. Application of artificial neural networks for Process Analytical Technology-based dissolution testing. Int J Pharm 567, 118464. https://doi.org/10.1016/j.ijpharm.2019.118464

Närvänen, T., Antikainen, O., Yliruusi, J., 2009. Predicting Particle Size During Fluid Bed Granulation Using Process Measurement Data. AAPS PharmSciTech 10, 1268. https://doi.org/10.1208/s12249-0099310-6

Otsuka, M., Tanabe, H., Osaki, K., Otsuka, K., Ozaki, Y., 2007. Chemoinformetrical evaluation of dissolution property of indomethacin tablets by near-infrared spectroscopy. J Pharm Sci 96, 788-801. https://doi.org/10.1002/jps.20704 
1013 Palmer, J., O’Malley, C., Wade, M., Martin, E., Page, T., Montague, G., 2018. Opportunities for Process 1014 Control and Quality Assurance Using Online NIR Analysis to a Continuous Wet Granulation Tableting 1015 Line. J Pharm Innov 12, 1 15. https://doi.org/10.1007/s12247-018-9364-7

1016

1017

Paudel, A., Raijada, D., Rantanen, J., 2015. Raman spectroscopy in pharmaceutical product design. Adv

1018

Drug Deliver Rev 89, 3 20. https://doi.org/10.1016/j.addr.2015.04.003

1019

1020

1021

Pauli, V., Roggo, Y., Pellegatti, L., Trung, N., Elbaz, F., Ensslin, S., Kleinebudde, P., Krumme, M., 2019.

1022

Process analytical technology for continuous manufacturing tableting processing: A case study. J Pharm

1023

1024

Pawar, P., Wang, Y., Keyvan, G., Callegari, G., Cuitino, A., Muzzio, F., 2016. Enabling real time release

1025

testing by NIR prediction of dissolution of tablets made by continuous direct compression (CDC). Int J

1026

Pharm 512, 96 107. https://doi.org/10.1016/j.ijpharm.2016.08.033

1027

1028

Pérez-Ramos, J.D., Findlay, P.W., Peck, G., Morris, K.R., 2005. Quantitative analysis of film coating in a

1029

pan coater based on in-line sensor measurements. AAPS PharmSciTech 6, E127 E136.

1030

https://doi.org/10.1208/pt060120

1031

1032

Pestieau, A., Krier, F., Thoorens, G., Dupont, A., Chavez, P.-F., Ziemons, E., Hubert, P., Evrard, B., 2014.

1033

Towards a real time release approach for manufacturing tablets using NIR spectroscopy. J Pharm

1034

Biomed 98, 60 67. https://doi.org/10.1016/j.jpba.2014.05.002

1035

1036

Quodbach, J., Kleinebudde, P., 2015. A critical review on tablet disintegration. Pharm Dev Technol 21, 1

1037

12. https://doi.org/10.3109/10837450.2015.1045618

1038

1039

Razavi, S.M., Callegari, G., Drazer, G., Cuitiño, A.M., 2016. Toward predicting tensile strength of pharmaceutical tablets by ultrasound measurement in continuous manufacturing. Int J Pharm 507, 83 89. https://doi.org/10.1016/j.ijpharm.2016.04.064

1042

1043

Reich, G., 2005. Near-infrared spectroscopy and imaging: Basic principles and pharmaceutical

1044 applications. Adv Drug Deliver Rev 57, 1109 1143. https://doi.org/10.1016/j.addr.2005.01.020 
1046

1047

1048

1049

1050

1051

1052

1053

1054

1055

1056

1057

1058

1059

1060

1061

1062

1063

1064

1065

1066

1067

1068

1069

1070

1071

1072

1073

1074

1075

1076

1077

Rinnan, Åsmund, van den Berg, F., Engelsen, S., 2009. Review of the most common pre-processing techniques for near-infrared spectra. Trac Trends Anal Chem 28, 1201-1222.

https://doi.org/10.1016/j.trac.2009.07.007

Roggo, Y., Chalus, P., Maurer, L., Lema-Martinez, C., Edmond, A., Jent, N., 2007. A review of near infrared spectroscopy and chemometrics in pharmaceutical technologies. J Pharm Biomed 44, 683700. https://doi.org/10.1016/j.jpba.2007.03.023

Römer, M., Heinämäki, J., Miroshnyk, I., Kivikero, N., Sandler, N., Rantanen, J., Yliruusi, J., 2008. Phase Transformation of Erythromycin A Dihydrate During Fluid Bed Drying, J Pharm Sci 97, 4020-4029. https://doi.org/10.1002/jps.21272

Sacher, S., Wahl, P., Weißensteiner, M., Wolfgang, M., Pokhilchuk, Y., Looser, B., Thies, J., Raffa, A., Khinast, J.G., 2019. Shedding Light on Coatings: Real-time Monitoring of Coating Quality at Industrial Scale. Int J Pharm 566, 57-66. https://doi.org/10.1016/j.ijpharm.2019.05.048

Sandell, D., Vukovinsky, K., Diener, M., Hofer, J., Pazdan, J., Timmermans, J., 2006. Development of a Content Uniformity Test Suitable for Large Sample Sizes. Drug Inf J 40, 337-344.

https://doi.org/10.1177/009286150604000312

Schmidt-Lehr, S., Moritz, H.-U., Jurgens, K.C., 2007. Online Control of Particle Size during Fluidised Bed Granulation. Pharmazeutische Industrie 69, 478--484.

Shi, Z., Hermiller, J., Muñoz, S., 2019. Estimation of mass-based composition in powder mixtures using Extended Iterative Optimization Technology (EIOT). AIChE J 65, 87-98.

https://doi.org/10.1002/aic.16417

Sibik, J., Chalus, P., Maurer, L., Murthy, A., Krimmer, S., 2017. Mechanistic approach in powder blending PAT: Bi-layer mixing and asymptotic end point prediction. Powder Technol 308, 306-317. https://doi.org/10.1016/j.powtec.2016.12.038

Sjöholm, M., Somesfalean, G., Alnis, J., Andersson-Engels, S., Svanberg, S., 2001. Analysis of gas dispersed in scattering media. Opt Lett 26, 16-18. https://doi.org/10.1364/OL.26.000016 
1078

1079

1080

1081

1082

1083

1084

1085

1086

1087

1088

1089

1090

1091

1092

1093

1094

1095

1096

1097

1098

1099

1100

1101

1102

1103

1104

1105

1106

1107

1108

1109

1110

1111

Svensson, T., Persson, L., Andersson, M., Svanberg, S., Andersson-Engels, S., Johansson, J., Folestad, S., 2007. Noninvasive characterization of pharmaceutical solids by diode laser oxygen spectroscopy, Appl Spectrosc 61, 784-786.

Tabasi, S., Fahmy, R., Bensley, D., O’Brien, C., Hoag, S.W., 2008a. Quality by design, part II: Application of NIR spectroscopy to monitor the coating process for a pharmaceutical sustained release product. J

Pharm Sci 97, 4052-4066. https://doi.org/10.1002/jps.21307

Tabasi, S., Fahmy, R., Bensley, D., O’Brien, C., Hoag, S.W., 2008b. Quality by design, part I: Application of NIR spectroscopy to monitor tablet manufacturing process. J Pharm Sci 97, 4040-4051.

https://doi.org/10.1002/jps.21303

USP, 2011. <905> Uniformity of Dosage Units.

Vervaet, C., Remon, J., 2005. Continuous granulation in the pharmaceutical industry. Chem Eng Sci 60, 3949-3957. https://doi.org/10.1016/j.ces.2005.02.028

Viljoen, J.M., Steenekamp, J.H., Marais, A.F., Kotzé, A.F., 2013. Effect of moisture content, temperature and exposure time on the physical stability of chitosan powder and tablets. Drug Dev Ind Pharm 40, 730-742. https://doi.org/10.3109/03639045.2013.782501

Vogt, F. G., Kord A. S., 2011. Development of Quality-By-Design Analytical Methods, J Pharm Sci 100, 797-812. https://doi.org/10.1002/jps.22325

Wahl, P.R., Fruhmann, G., Sacher, S., Straka, G., Sowinski, S., Khinast, J.G., 2014. PAT for tableting: Inline monitoring of API and excipients via NIR spectroscopy. Eur J Pharm Biopharm 87, 271278.

https://doi.org/10.1016/j.ejpb.2014.03.021

Ward, H.W., Blackwood, D.O., Polizzi, M., Clarke, H., 2013. Monitoring blend potency in a tablet press feed frame using near infrared spectroscopy. J Pharm Biomed 80, 1823.

https://doi.org/10.1016/j.jpba.2013.02.008

Whitaker, M., Baker, G.R., Westrup, J., Goulding, P.A., Rudd, D.R., Belchamber, R.M., Collins, M.P., 2000. Application of acoustic emission to the monitoring and end point determination of a high shear 
1112 granulation process. Int J Pharm 205, 79 91. https://doi.org/10.1016/s0378-5173(00)00479-8

1114 Wikströmm H., Marsac, P. J., Taylor, L. S., 2005. In-Line Monitoring of Hydrate Formation during Wet

1115 Granulation Using Raman Spectroscopy, J Pharm Sci 94, 209-219. https://doi.org/10.1002/jps.20241

1116

1117 Wirges, M., Funke, A., Serno, P., Knop, K., Kleinebudde, P., 2013. Development and in-line validation of a

1118 Process Analytical Technology to facilitate the scale up of coating processes. J Pharm Biomed 78, 57-64.

1119 https://doi.org/10.1016/j.jpba.2013.01.037

1120

1121 Wold, S., Sjöström, M., Eriksson, L., 2001. PLS-regression: a basic tool of chemometrics. Chemometr

1122 Intell Lab 58, 109-130. https://doi.org/10.1016/s0169-7439(01)00155-1

1123

1124 Yu, L.X., Amidon, G., Khan, M.A., Hoag, S.W., Polli, J., Raju, G., Woodcock, J., 2014. Understanding

1125 Pharmaceutical Quality by Design. AAPS J 16, 771-783. https://doi.org/10.1208/s12248-014-9598-3

1126

1127 Zaborenko, N., Shi, Z., Corredor, C.C., ith-Goettler, B., Zhang, L., Hermans, A., Neu, C.M., Alam, M.,

1128 Cohen, M.J., Lu, X., Xiong, L., Zacour, B.M., 2019. First-Principles and Empirical Approaches to Predicting

1129 In Vitro Dissolution for Pharmaceutical Formulation and Process Development and for Product Release

1130 Testing. AAPS J 21, 32. https://doi.org/10.1208/s12248-019-0297-y

1131

1132 Zannikos, P.N., Li, W.-I., Drennen, J.K., Lodder, R.A., 1991. Spectrophotometric Prediction of the

1133 Dissolution Rate of Carbamazepine Tablets. Pharm Res 8, 974-978.

1134 https://doi.org/10.1023/a:1015840604423 


\section{Figure Captions}

1137 Figure 1: Process flow diagram for direct compression, dry granulation and wet granulation highlighting 1138 the points of testing and properties of interest. S01 - S09 refer to points of testing (sensor positions) in 1139 the process line. Red text refers to CQAs.

1140 Figure 2: Overview of CQAs and measurement techniques. NIR: near-infrared spectroscopy; Raman: 1141 Raman spectroscopy; MRT: microwave resonance technology, DFF: drag force flow sensor; PIT: 1142 photometric imaging technique; $\mathrm{AE}$ : acoustic emission; SFV: spatial filter velocimetry; TDS: terahertz time1143 domain spectroscopy; HWT: hardness, weight, thickness; TPI: terahertz pulsed imaging; OCT: optical 1144 coherence tomography; GASMAS: gas in scattering media absorption spectroscopy.

1145 Figure 3: RTRT development workflow. RTD: residence time distribution.

1146 Figure 4: Building blocks of the RTR monitoring and control strategy.

1147 Figure 5: Tablet weight versus API concentration collection limits example. 
1150 Table 1: Points of testing for powder, blend, ribbons, granules, tablet cores and coated tablets. The sensor 1151 reference denotes the sensor position in Figure 1.

1152 Table 2: RTRT use case from AstraZeneca.

1153 Table 3: RTRT use case from Eli Lilly. The combination of blend and tablet uniformity are used to control 1154 tablet content uniformity.

1155 Table 4: Summary of literature of RTRT application in tablet manufacturing. Measurement time: Time for individual measurements (integration time and averaging included). R: Reflection; T: Transmission; NA: not applicable; NP: not provided; $R^{2}$ : coefficient of determination; $Q^{2}$ : goodness of prediction; $R M S E$ : root mean squared error; RMSEC: root mean squared error calibration; RMSEP: root mean squared error prediction; RMSECV: root mean squared error cross-validation; SEC: standard error calibration; SECV: standard error cross-validation; EIOT: extended iterative optimization technique; NIR: near-infrared spectroscopy; Raman: Raman spectroscopy; MRT: microwave resonance technology, DFF: drag force flow sensor; PIT: photometric imaging technique; AE: acoustic emission; SFV: spatial filter velocimetry; TDS: terahertz time-domain spectroscopy; HWT: hardness, weight, thickness; TPI: terahertz pulsed imaging; OCT: optical coherence tomography. 